\title{
Apprécié Exil : Un service de réhabilitation pour les victimes de tortures
}

Le Centre Exil

\section{Le Centre Exil : Depuis plus de 30 ans}

耳xil est un centre de santé mentale situé à Bruxelles (Belgique) Eet spécialisé dans la réhabilitation de réfugiés ayant été victimes de tortures et/ou de violence organisée dans leur pays d'origine. Le Centre Exil trouve ses racines dans l'expérience du COLAT (Collectif Latino-Américain de Travail Psycho-social) fondé en 1976 par des professionnels de la santé latino-américains qui, ayant été eux-mêmes victimes de violations des Droits de l'Homme dans leurs pays d'origine, ont réussi à s'organiser, avec le soutien de professionnels européens, pour mettre leurs expériences et compétences professionnelles au service de leur communauté d'exilés. Ainsi, ils ont développé un programme de santé communautaire destiné à prévenir et traiter les effets de la violence organisée et des multiples violations des Droits de l'Homme chez l'individu, la famille et la communauté produits par les dictatures latino-américaines.

L'équipe est constituée de professionnels de disciplines et cultures différentes et offre ainsi aux personnes qui consultent un cadre médico-psycho-social interdisciplinaire et interculturel. L'approche de la souffrance est globale et les soins sont donnés par une équipe de professionnels multidisciplinaire composée de médecins généralistes, de psychiatres, de psychologues, de travailleurs sociaux et d'un personnel d'accueil. L'approche thérapeutique est invariante dans son éthique mais elle a été confrontée de façon permanente aux politiques locales et internationales de réglementation des flux migratoires et de la politique d'asile.

Depuis sa création, le COLAT a bénéficié de l'appui et du soutien académique du Département de psychiatrie de la Katholieke Universiteit van Leuven, dirigé par le professeur Franz Baro. De plus, le projet a été, depuis ses débuts jusqu'à l'année 1984, soutenu financièrement par des institutions non-gouvernementales européennes (Brott fur die Welt, en Allemagne, le Conseil Mondial des Églises, en Suisse, Entraide et Fraternité et Broederlijk Delen, en Belgique) et par des organismes internationaux (Le Haut Commissariat pour les Réfugiés des Nations Unies, et plus tard, le Fonds Volontaire des Nations Unies pour les victimes de la torture). 


\section{LE PROGRAMME THÉRAPEUTIQUE DE RÉHABILITATION DES VICTIMES DE TORTURES}

Depuis près de trente ans, le Centre Exil développe un programme spécifique d'accompagnement global de la santé pour des personnes exilées et victimes de tortures. Bénéficiant d'une expérience avec plusieurs dizaines de milliers de patients, les modèles d'intervention ont été affinés au cours des années. Toutes les victimes de tortures qui consultent le Centre ont la possibilité de bénéficier d'une psychothérapie individuelle et/ou de groupe à long-terme.

Un dialogue avec les psychothérapeutes et les différents membres du groupe sur les enjeux de la situation vécue est destiné à aider la victime à se resituer par rapport aux techniques de manipulation psychologique des tortionnaires. Ce modèle d'intervention a pour finalité d'aider les victimes à se libérer de l'emprise psychologique des tortionnaires en découvrant le processus de culpabilisation auquel elles ont été soumises par les bourreaux et en encadrant à nouveau leur vécu de peur, de doutes quant à leurs valeurs personnelles, de honte et de souffrance, comme étant des réponses naturelles face à la situation extrême. Dans ce contexte, la victime peut prendre conscience de ses ressources personnelles et réaliser comment sa propre créativité lui a permis de survivre. Toutes les démarches thérapeutiques s'adressent à l'individu en relation avec son entourage familial et social.

Le modèle d'intervention et les techniques thérapeutiques ont pour finalité d'agir sur:

1) L'aspect traumatique : conséquence de la douleur extrême, les dommages corporels, la perte des camarades, amies et/ou membres de la famille, les violences sexuelles, parmis d'autres blessures subis par les victimes. Dès lors, il s'agit de soigner les blessures somatiques et psychologiques provoquées par la torture.

2) Le traumatisme de la personnalité, résultat du processus de manipulation psychologique des tortionnaires. Il s'agit ici de guérir le processus d'aliénation de la personnalité des victimes et les conséquences du processus de lavage de cerveau.

3) L'infiltration de l'horreur dans le réseau familial et social 
des victimes. À ce niveau, l'intervention se situe autour de la reconstruction de la famille et du tissu social de la victime.

Parmi tous les patients qui consultent le Centre Exil, les enfants sont les plus vulnérables car ils cumulent cette série de facteurs de fragilisation. Mais la perte la plus importante vécue par l'enfant victime de la guerre est celle de sa sécurité de base et de sa confiance dans les adultes, qui au lieu de lui apporter soutien et protection, ont été les acteurs du drame qui a ravagé son existence. De plus, la souffrance et le déracinement vécus par les parents provoquent une situation de vulnérabilité dans l'exercice de la fonction parentale et induit un risque plus important de maltraitance et de négligence. Notre action porte donc sur l'enfant, mais aussi sur ses parents, ainsi que les autres adultes qui l'entourent. Nous suivons également des adolescents non-accompagnés ou vivant en famille, avec des membres de leur communauté ou d'autres personnes les ayant accueillis dans leur foyer. L'objectif principal de nos interventions est de soutenir leur recherche d'identité et d'autonomie dans le pays qui les accueille. Il s'agit également de soutenir ces jeunes qui vivent de profondes difficultés liées à la négligence ou l'absence parentale. La fragilisation des femmes met en risque la structure identitaire individuelle et culturelle de chaque sujet. Notre équipe est préoccupée par l'état de santé de ces femmes et considère que ce projet permet, dans la continuité qui est proposée, d'éviter la chronicisation de leur souffrance et leur exclusion de la société. En ce qui concerne les hommes, il s'agit de leur offrir un traitement médical, psychiatrique, psychologique de nature à leur permettre de retrouver un bien-être et à proposer des activités communautaires de manière à les sortir de la solitude.

Parallèlement aux activités thérapeutiques en Belgique, les professionnels du COLAT ont participé de façon active à la restauration des Droits de l'Homme et de la démocratie dans différents pays latinoaméricains, ainsi qu'au soutien de l'action des différentes organisations non-gouvernementales locales, qui, spécialement au Chili, en Uruguay, en Argentine et en Amérique Centrale, travaillaient pour la défense des Droits de l'Homme et le soutien médico-psycho-social des victimes sur place. Différents membres de l'équipe thérapeutique du COLAT ont participé à des missions clandestines à l'intérieur de ces pays pour soutenir ces combats. Depuis janvier 1985, le COLAT a commencé à élargir ses services à d'autres communautés de réfugiés provenant de différents pays du monde. En effet, suivant les événements socio-politiques, ce sont d'autres victimes de violations des Droits de l'Homme qui demandent l'asile en Belgique. C'est ainsi qu'en 1986, le Centre COLAT devient le Centre EXIL (Centre médico-psycho-social pour Réfugiés et Victimes 
de Tortures) en ouvrant ses différents services à toutes les communautés de réfugiés et en prenant en charge de façon progressive le suivi médicopsycho-social des demandeurs d'asile et d'une partie importante des personnes déboutées par la procédure de reconnaissance du statut de réfugié. Aujourd'hui les personnes victimes de violations des Droits de l'Homme qui bénéficient du Programme d'Exil sont originaires d'Afrique, d'Amérique Latine, d'Asie, du Moyen-Orient et d'Europe de l'Est. Ces personnes, en plus d'avoir connu toutes sortes de violations de leurs droits et d'avoir été victimes de situations de violence extrême, sont confrontées à une dégradation des conditions d'accueil. Ainsi, un des axes du programme d'Exil est de promouvoir des conditions d'accueil favorables dans la société belge. En effet, nos expériences cliniques nous ont appris qu'un accueil solidaire est un des facteurs fondamentaux pour soutenir les ressources naturelles des victimes de violence dans leur combat pour reconstruire leur identité ainsi que leur réseau familial et social, et ainsi dépasser les traumatismes consécutifs à la répression, l'emprisonnement, la torture, le viol, la mort des proches, parmi plusieurs autres chocs et construire un projet d'avenir.

\section{Pour Plus de Renseignements}

Le Centre Exil 282 Avenue de la Couronne 1050 Bruxelles, Belgique

Téléphone : +3225345330 Fax : +32 25349016

E-mail : info@exil.be Site web:www.exil.be 